\title{
Enzymatic- and temperature-sensitive controlled release of ultrasmall superparamagnetic iron oxides (USPIOs)
}

\author{
Shann S Yu ${ }^{1,2}$, Randy L Scherer ${ }^{2,3}$, Ryan A Ortega ${ }^{1,2}$, Charleson S Bell ${ }^{1,2}$, Conlin P O'Neil ${ }^{4}$, Jeffrey A Hubbell ${ }^{4}$, \\ Todd D Giorgio ${ }^{1,2^{*}}$
}

\begin{abstract}
Background: Drug and contrast agent delivery systems that achieve controlled release in the presence of enzymatic activity are becoming increasingly important, as enzymatic activity is a hallmark of a wide array of diseases, including cancer and atherosclerosis. Here, we have synthesized clusters of ultrasmall superparamagnetic iron oxides (USPIOs) that sense enzymatic activity for applications in magnetic resonance imaging (MRI). To achieve this goal, we utilize amphiphilic poly(propylene sulfide)-bl-poly(ethylene glycol) (PPS-b-PEG) copolymers, which are known to have excellent properties for smart delivery of drug and siRNA.

Results: Monodisperse PPS polymers were synthesized by anionic ring opening polymerization of propylene sulfide, and were sequentially reacted with commercially available heterobifunctional PEG reagents and then ssDNA sequences to fashion biofunctional PPS-bl-PEG copolymers. They were then combined with hydrophobic $12 \mathrm{~nm}$ USPIO cores in the thin-film hydration method to produce sSDNA-displaying USPIO micelles. Micelle populations displaying complementary ssDNA sequences were mixed to induce crosslinking of the USPIO micelles. By design, these crosslinking sequences contained an EcoRV cleavage site. Treatment of the clusters with EcoRV results in a loss of $R_{2}$ negative contrast in the system. Further, the USPIO clusters demonstrate temperature sensitivity as evidenced by their reversible dispersion at $\sim 75^{\circ} \mathrm{C}$ and re-clustering following return to room temperature.

Conclusions: This work demonstrates proof of concept of an enzymatically-actuatable and thermoresponsive system for dynamic biosensing applications. The platform exhibits controlled release of nanoparticles leading to changes in magnetic relaxation, enabling detection of enzymatic activity. Further, the presented functionalization scheme extends the scope of potential applications for PPS-b-PEG. Combined with previous findings using this polymer platform that demonstrate controlled drug release in oxidative environments, smart theranostic applications combining drug delivery with imaging of platform localization are within reach. The modular design of these USPIO nanoclusters enables future development of platforms for imaging and drug delivery targeted towards proteolytic activity in tumors and in advanced atherosclerotic plaques.
\end{abstract}

\section{Background}

Enzymatic activity is understood to be a hallmark of various diseases, including cancer and atherosclerosis [1,2]. Consequently, enzymatically-sensitive drug- and contrast agent-delivery platforms are of great interest in medical areas. Enzymatically-sensitive controlled release platforms have been previously investigated for drug

\footnotetext{
* Correspondence: todd.d.giorgio@vanderbilt.edu

'Department of Biomedical Engineering, Vanderbilt University; Nashville, Tennessee, USA

Full list of author information is available at the end of the article
}

delivery [3-5]. While they have also been investigated for molecular imaging, most of these efforts have been concentrated in the areas of optical imaging and nuclear imaging $[1,6,7]$. In many cases, these techniques are disadvantageous for in vivo applications because optical imaging is significantly limited by tissue autofluorescence and light absorbance, while nuclear imaging can expose the patient to relatively high doses of ionizing radiation. Magnetic resonance imaging (MRI) is not limited by these issues and provides the advantages of high spatial resolution and excellent soft tissue contrast. Only

\section{Biomed Central}


a few examples of enzymatically-sensitive platforms for MRI applications have been previously reported, as reviewed elsewhere [1].

Ultrasmall superparamagnetic iron oxides (USPIOs) have been widely investigated for applications as MRI contrast agents and for probing intermolecular interactions due to their strong T2 magnetic relaxation properties [8-11]. As contrast agents, USPIOs have unique characteristics, including high detection sensitivity, relatively low toxicity, and the potential for long circulation half-lives $[12,13]$. To produce USPIOs of uniform composition, size, and physical properties, thermal decomposition synthesis is preferred, but the process yields USPIO cores coated with a layer of the hydrophobic surfactant oleic acid [14].

Especially for our applications, biocompatible, bioactive USPIO-based contrast agents must exhibit solubility and stability in water and, in many cases, to display ligands such as whole proteins, peptides, or nucleic acids. In order to achieve this goal, a modular approach for functionalizing USPIOs is generally followed. Various methods for rendering USPIOs water-soluble are welldocumented, including covalent methods such as silanization or the formation of micelles with polymers or phospholipids [8,15-18]. A wide range of techniques in bioconjugate chemistry can then be used to immobilize bioactive ligands onto the USPIO surface [19].

Some USPIO formulations are biocompatible and have been clinically approved for human use, such as Feridex and GastroMARK [20-22]. However, nanoparticle biocompatibility is largely determined by surface properties, independent of USPIO characteristics. Because of this, in vivo biodistribution must be determined for each unique formulation [23-26].

In recent years, the encapsulation of USPIOs in micellar structures by self-assembly with amphiphilic PEGcontaining block copolymers has received attention $[17,27,28]$. Recently, extensive studies by the Hubbell group have shown that amphiphilic block copolymers of PEG and the hydrophobic poly(propylene sulfide) (PPS) can be used to generate micellar and multilamellar structures for drug delivery applications [29,30]. These copolymers have received interest for their unique characteristics, including a PPS block capable of undergoing a hydrophobic-to-hydrophilic transition in oxidative environments, resulting in environmentally-sensitive drug release $[30,31]$. Though previously uninvestigated as a USPIO coating, the PEG-PPS copolymers display material properties that presumably enable the construction of novel oxidation-responsive "theranostic" (therapeutic-diagnostic) agents in the near future. To add to these properties, PEG-PPS copolymers have been successfully tagged with bioactive ligands such as peptides for actively targeted drug delivery [32]. Here, we report the broader utility of the PEG-PPS copolymer platform through the synthesis of PPS-PEG-ssDNA constructs, and the self-assembly of these constructs onto highly monodisperse USPIO cores to generate multifunctional magnetofluorescent nanoparticles.

To demonstrate the applicability of the approach, these novel ssDNA-tagged USPIOs will then be assessed as magnetic relaxation switches (MRS) [33]. The MRS concept indicates that clustering of USPIOs leads to a significant increase in $\mathrm{R}_{2}$ relaxivity of the USPIOs, while redispersion of the USPIOs returns $R_{2}$ to baseline levels. The MRS label originated from the behavior of the system as a nanosensor capable of being turned on or off in the presence of a specific environmental stimulus, which, in this study, is restriction enzyme activity. Complementary populations of ssDNA-USPIOs were mixed to form self-assembled clusters. These clusters were subjected to restriction enzyme treatment or thermocycling to exert controlled release of the USPIO cores. Light scattering and relaxation measurements were carried out on clustered and declustered MRS in aqueous solution. The work presented here offers a flexible platform for generating biocompatible, MR-visible nanomaterials with $\mathrm{T} 2$ relaxivities modulated by enzyme activity that presumably enable in vivo biosensing by modulation of image contrast.

\section{Results and Discussion}

\section{Synthesis of PEG-PPS block copolymers and encapsulation of USPIO cores}

The anionic ring opening polymerization scheme allows some degree of flexibility in fashioning PPS blocks with various functional groups on both ends of the polymer chain. This is done by varying the initiator and the chain terminator used in the reaction [34-38]. Ethanethiol was chosen as an initiator because the thiol is easily deprotonated by a small excess of DBU, without significant risk of the DBU leading to side products during the polymerization process (Figure 1). Injection of a stoichiometric amount of the propylene sulfide monomer forms the PPS block, and the reaction is endcapped with methyl acrylate via a Michael-type addition mechanism [35]. Hydrolysis of the terminal methyl ester under alkaline conditions fashioned PPS-COOH, at $1.65 \mathrm{kDa}$ and PDI 1.18 (Table 1, Figure 2a). Acrylic acid was also investigated as an endcapping agent for the living polymerization in an attempt to fashion PPS-COOH in a single step, but resulted in undesirable side products that were likely formed by competing mechanisms to Michael-type addition (data not shown). Attachment of the PEG block and the ssDNA block were both done via well-characterized carbodiimide chemistry [19]. The construction of carboxylated PEG-PPS (cPEG-PPS) was confirmed via FT-IR (Figure 2b) and NMR 


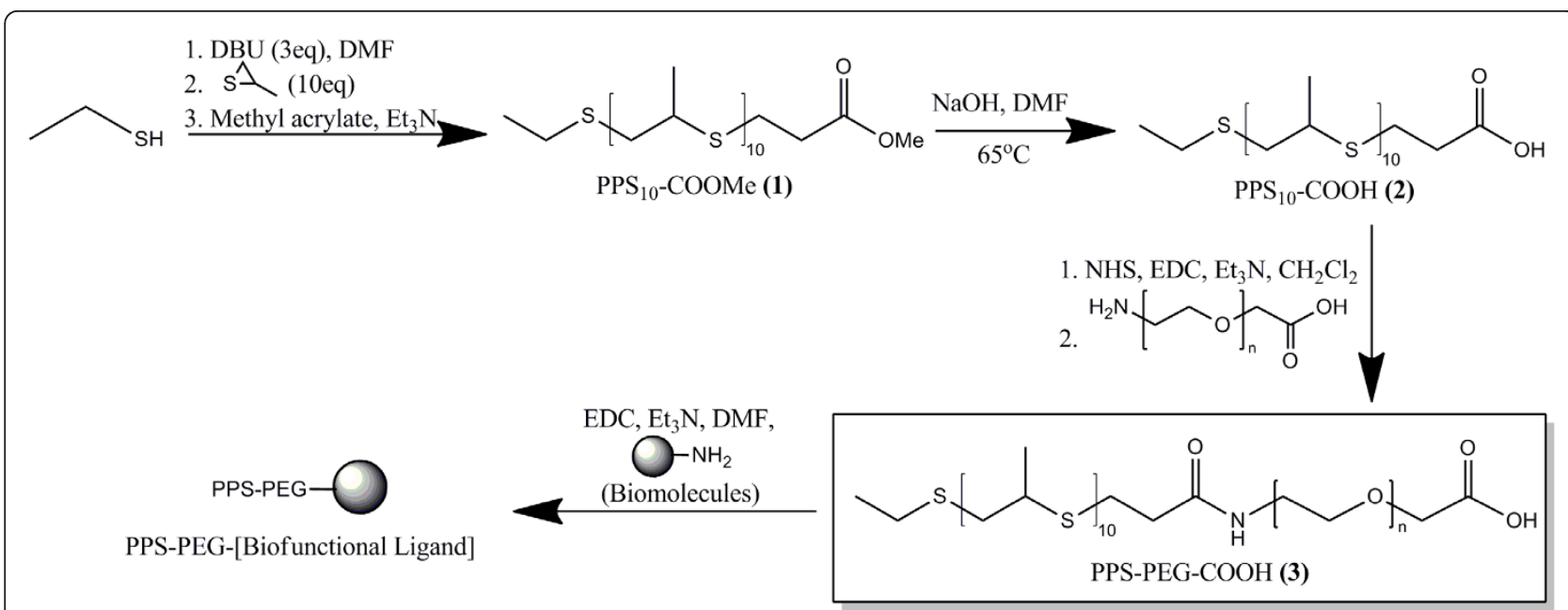

Figure 1 Synthesis of PEG-PPS-based polymer-biomolecule conjugates. The PPS block is formed by anionic ring-opening polymerization of an episulfide monomer, and endcapped with methyl acrylate. Conversion of the terminal methyl ester group to a carboxylic acid is accomplished under highly basic conditions to enable subsequent coupling of a PEG block and then a biofunctional ligand (e.g., peptides, amine-functionalized ssDNA) in modular fashion, yielding PEG-PPS-based polymer-biomolecule conjugates.

spectroscopies, and further visualized by GPC, as the copolymers exhibited an elution peak centered at around 9 min that was not seen on the elution profiles of either of the precursor blocks (Figure 2a). Attachment of the ssDNA block was confirmed by UV-Vis spectrophotometry of extensively dialyzed PPS-PEG-ssDNA, characterized by the appearance of a peak at $260 \mathrm{~nm}$ for both ssDNA-coupled samples (Figure 2c).

With the PEG-PPS copolymers and the polymerssDNA conjugates complete, polymer-coated USPIOcore micelles were formed using the thin film hydration method [39]. In this process, a mixture of as-synthesized USPIO cores and polymers in toluene is completely dried by rotary evaporation, and then rehydrated to form micelles. In concept, the hydrophobic PPS blocks are expected to mingle with the oleic acid surfactant on the USPIO surface, with the PEG blocks and ssDNA extending into the surrounding aqueous medium, stabilizing the micelle. The micellization process resulted in a considerable amount of insoluble side products that can be easily precipitated away by magnet, leaving a colloidal phase that is then isolated into a fresh vial. Free, unloaded PEG-PPS was colloidally unstable and was easily removed by centrifugation, but the ironcontaining micelles appeared stable in water and did not flocculate over several months. As little as 1.5:1 (w/w) ratio of polymer to iron oxide is sufficient to render PEG-PPS-USPIO micelles water-soluble. The micelles exhibited hydrodynamic diameters of $41 \mathrm{~nm}$ as measured by DLS. They appear so colloidally stable that they are extremely difficult to pellet without an ultracentrifuge, and are very slowly pelleted in proximity to a $1 \mathrm{~T}$-field strength neodymium magnet. These observations have been suggested by other groups working with colloidal USPIOs [40-42].

The morphology of the particles before and after encapsulation in PEG-PPS was assessed by TEM. Asmade oleic acid-stabilized $12 \pm 1 \mathrm{~nm}$ USPIO cores were deposited and dried on the TEM grid from toluene and generally appeared well-dispersed, but were also capable of forming short-ranged packing structures that showcased their monodispersity (Figure 3a). These same

Table 1 Molecular weight data for synthesized polymers ${ }^{a)}$

\begin{tabular}{|c|c|c|c|c|c|c|}
\hline \multirow[t]{2}{*}{ Polymers } & $\mathrm{dn} / \mathrm{dc}$ at $40^{\circ} \mathrm{C}^{\mathrm{b})}$ & $M_{n}$ & $M_{w}$ & PDI & $M_{n}$ from NMR & \multirow[t]{2}{*}{ Average Degree of polymerization by NMR } \\
\hline & $\mathrm{mL} / \mathrm{g}$ & $\mathrm{Da}$ & $\mathrm{Da}$ & unitless & Da & \\
\hline PPS-COOH & 0.246 & 1650 & 1950 & 1.18 & 874 & 10 \\
\hline $\mathrm{H}_{2} \mathrm{~N}-\mathrm{PEG}-\mathrm{COOH}{ }^{\mathrm{c})}$ & - & 4200 & - & - & - & 110 \\
\hline cPEG-PPS & 0.183 & 7320 & 10200 & 1.39 & 5100 & - \\
\hline
\end{tabular}

a) Molecular weight of polymers was determined by GPC-MALS. Polymers were injected into a TSKGel Mixed Bed HZM-N column (4.6 mm ID $\times 15 \mathrm{~cm}$ ) and chromatograms from the MALS detector and differential refractive index detector were used to analyze for polydispersity.

b) $\mathrm{dn} / \mathrm{dc}$ values were measured in offline batch mode by direct injection of serial dilutions of polymer samples into the refractive index detector of the GPC. The sample cell was maintained at $40^{\circ} \mathrm{C}$. Data analysis was done on the Wyatt Astra software.

c) Values for $M_{n}, M_{w}$, and PDI were provided by manufacturer. 


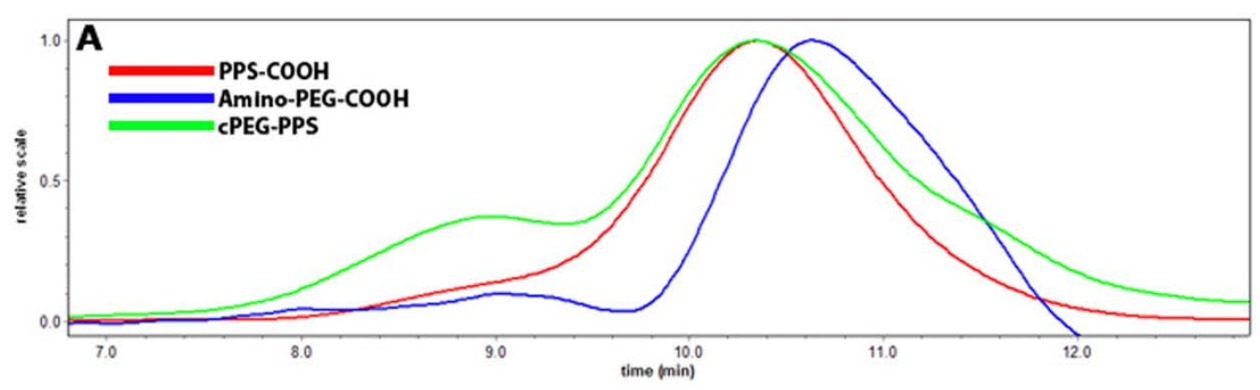

B)

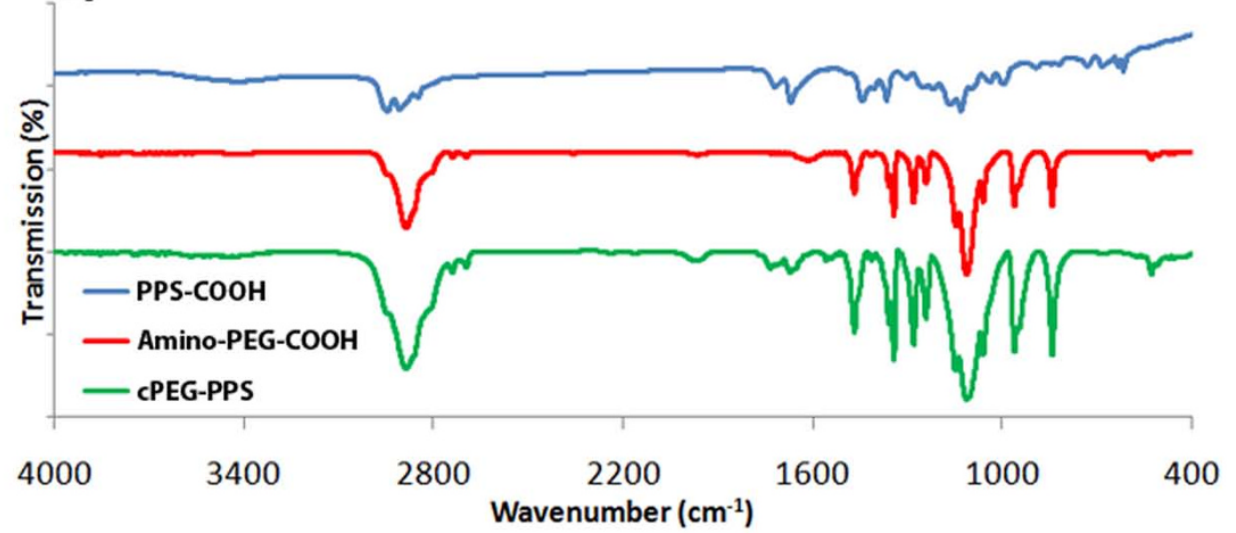

C)

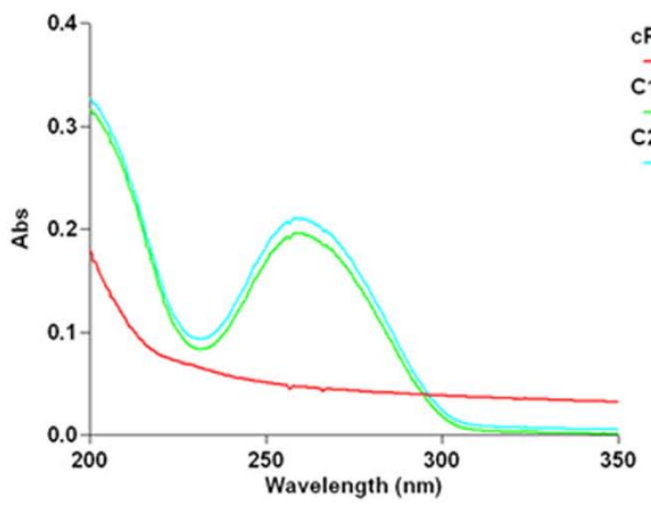

Figure 2 Characterization of PEG-PPS copolymers. (A) GPC-MALS characterization of PPS-COOH (red), $\mathrm{H}_{2} \mathrm{~N}-\mathrm{PEG}-\mathrm{COOH}$ (blue), and cPEG-PPS (green). The CPEG-PPS sample displayed a population of polymers with peak elution time at $\sim 9 \mathrm{~min}$, corresponding to $\mathrm{M}_{\mathrm{n}}=7.32 \mathrm{kDa}$ (see Table 1), in addition to excess unreacted $\mathrm{H}_{2} \mathrm{~N}-\mathrm{PEG}-\mathrm{COOH}$. (B) FT-IR spectra of the same three polymer samples confirm the formation of the copolymer. The appearance of the 1700-1630 $\mathrm{cm}^{-1}$ peak and disappearance of the $1650-1590 \mathrm{~cm}^{-1}$ free amine bending peak in the copolymer versus the unreacted PEG is consistent with the formation of an amide linkage between the N-terminus of the PEG block and the C-terminus of the PPS block. (C) UV-Vis absorbance of cPEG-PPS (red) versus the post-dialyzed ssDNA-PEG-PPS conjugates (green, blue) confirms the conjugation of ssDNA to the polymers, as referenced by the characteristic peak at $260 \mathrm{~nm}$.

observations applied for the cPEG-PPS encapsulated USPIO micelles deposited and dried on the same copper TEM grids out of water (Figure $3 \mathrm{~b}$ ). The addition of PPS-PEG-ssDNA conjugates into the micellization process immobilized ssDNA on the USPIOs and also produced micelles of similar morphology (Figure 3c-d). These two populations of ssDNA-displaying USPIOs can be then mixed to form longer-range clusters that can be characterized by both TEM (Figure 3e) and DLS (Figure 3f). As shown in Figure 3f, free ssDNA-displaying USPIO micelles exhibited hydrodynamic diameters of $\sim 70 \mathrm{~nm}$ (30 nm increase from the diameter exhibited by PEG-PPS-USPIO micelles is easily attributable to the length of the immobilized ssDNA sequences), while the clusters display diameters of upwards of $1 \mu \mathrm{m}$. The $100-200 \mathrm{~nm}$ peak picked up by the DLS is attributable 

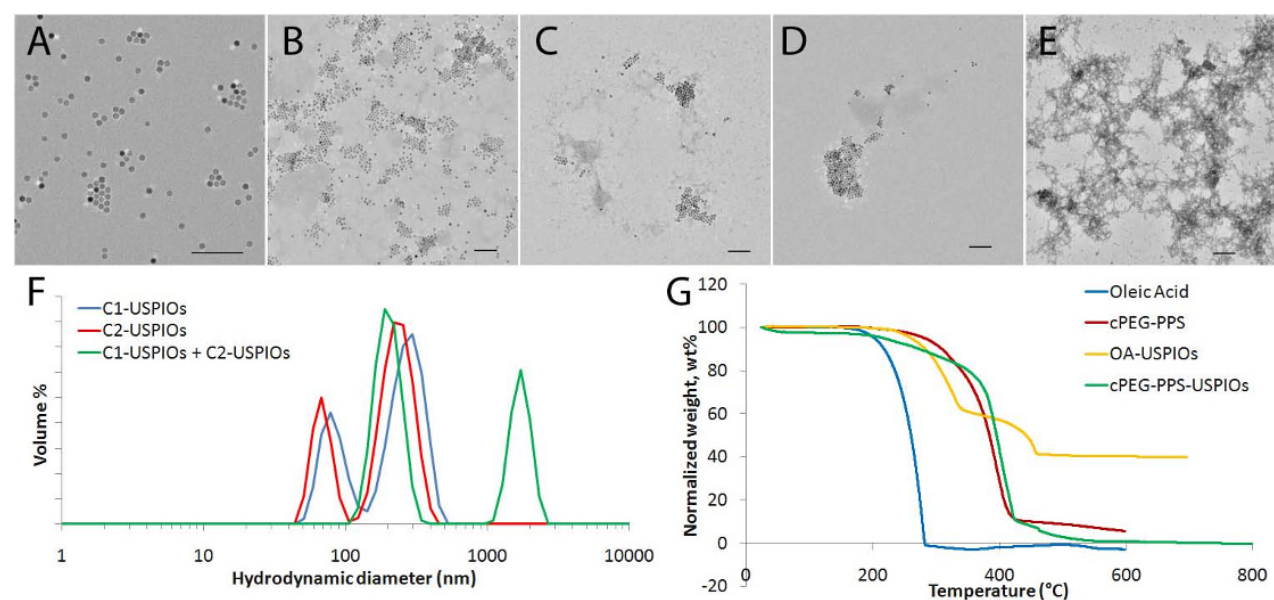

Figure 3 Properties of functionalized USPIOs. TEM of (A) as-made oleic acid-stabilized USPIO cores, (B) CPEG-PPS-USPIO micelles, (C) C1USPIOs, (D) C2-USPIOs, and (E) clusters formed by hybridization of C1-USPIOs and C2-USPIOs. All scale bars are in $100 \mathrm{~nm}$. In the first four cases, nanoparticles appear to be generally well-dispersed, but were also capable of forming short-ranged packing structures. (F) DLS size-volume distributions of C1-USPIOs (blue), C2-USPIOs (red), and clusters formed by hybridization of C1- and C2-USPIOs (green) suggest that the individual ssDNA-displaying USPIO micelles exhibit a hydrodynamic diameter of approximately $70 \mathrm{~nm}$, while clusters formed by mixing the two populations are generally greater than of $1 \mu \mathrm{m}$ in diameter. (G) TGA weight loss curves of oleic acid (blue), cPEG-PPS (red), OA-USPIOs (yellow), and CPEG-PPS-USPIOs (green) suggest that oleic acid is not displaced in the micellization process, and instead is encapsulated into the interior of the micelles along with the iron oxide core.

to excess, unloaded non-iron-containing PEG-PPS micelles that remain present in the samples, even following several centrifugation steps as described above.

The presence of the PEG-PPS coating was investigated by thermogravimetric analysis (TGA) (Figure 3g). The precursor OA-USPIOs displayed evaporation profiles corresponding to oleic acid $\left(250-350^{\circ} \mathrm{C}\right)$ and components of the iron oxide core $\left(350-450^{\circ} \mathrm{C}\right)$. It is unclear why USPIO-associated oleic acid evaporated later than free oleic acid, although it is interesting to note that our results match those of other groups working on oleatestabilized USPIOs [43]. cPEG-PPS-USPIO micelles also displayed two weight loss temperature ranges. The sharp evaporation range at $390-410^{\circ} \mathrm{C}$ can be attributed to the cPEG-PPS, while the long, gradual $200-390^{\circ} \mathrm{C}$ weight loss range is very likely made up of a combination of oleic acid evaporation, early cPEG-PPS desorption, and evaporation of iron oxide core components. This data suggests that the oleic acid surfactant remains anchored on the iron oxide cores during the micellization process with PEG-PPS, rather than being displaced in a ligandexchange reaction. Taken together, this data suggests PEG-PPS-based copolymers and conjugates were capable of stably rendering water-soluble USPIOs displaying immobilized ligands to the surrounding environment.

\section{Clustering and de-clustering of complementary USPIOs leads to modulation of $R_{2}$ relaxivity coefficients}

$R_{2}$ coefficients were calculated based on measurements of USPIO iron content through the phenanthroline assay [44] and relaxation time measurements. For all polymer-USPIO micelle samples, $R_{2}$ values ranged between $400-500 \mathrm{mM}^{-1} \mathrm{~s}^{-1}$. These values were well within expected ranges, and are similar in order of magnitude to those recently measured by LaConte et al and Lee et al $[45,46]$. Differences in the absolute $R_{2}$ values reported are easily accounted for, since LaConte et al used USPIO cores of smaller diameters $(\sim 6 \mathrm{~nm})$, while Lee et al used USPIO cores that had been doped with other metals such as manganese.

When C1-USPIOs and C2-USPIOs were mixed, the hybridization of the surface-immobilized ssDNA sequences resulted in crosslinking of the USPIOs into larger clusters. This response is observed via an increase in hydrodynamic diameters from $\sim 70 \mathrm{~nm}$ to above $1 \mu \mathrm{m}$ (Figure 3f), and an increase in $R_{2}$ coefficient to $690 \pm$ $230 \mathrm{mM}^{-1} \mathrm{~s}^{-1}$. These effects of USPIO clustering on $R_{2}$ are consistent with previously published results by $\mathrm{Ai}$ et al. [28]. Despite these previous demonstrations of this phenomenon, the mechanisms behind this "MRS" effect remain largely unstudied and are the subject of an ongoing study in our group.

Since clustering of the complementary C1-USPIOs and C2-USPIOs resulted in expected changes in $\mathrm{R}_{2}$, the next goal was to determine whether reversal of the clustering process would likewise correspondingly reverse the observed increase in $R_{2}$. Irreversible and reversible 'declustering' of the USPIO complexes was achieved through enzymatic treatment and through thermocycling experiments, respectively. 
By design, the hybridization of USPIO-immobilized C1- and C2- sequences reveals an EcoRV blunt end cleavage site. Treatment of the clusters with EcoRV is thus expected to redisperse the individual micelles, resulting in irreversible return of the $R_{2}$ values to the baseline levels prior to the formation of the clusters. The rate of de-clustering is expected to be controlled by the relative concentrations of the enzyme and substrate. The 4-hour enzyme treatment in this proof-of-concept study allowed the de-clustering to go to completion. These expectations are confirmed by relaxometry data (Figure 4a), where a stable and significant difference in $\mathrm{R}_{2}(\mathrm{p}<0.05)$ is measured following treatment with EcoRV. The final $R_{2}$ values remained stable for several hours following enzymatic treatment, suggesting that the declustering of the USPIOs was irreversible. In

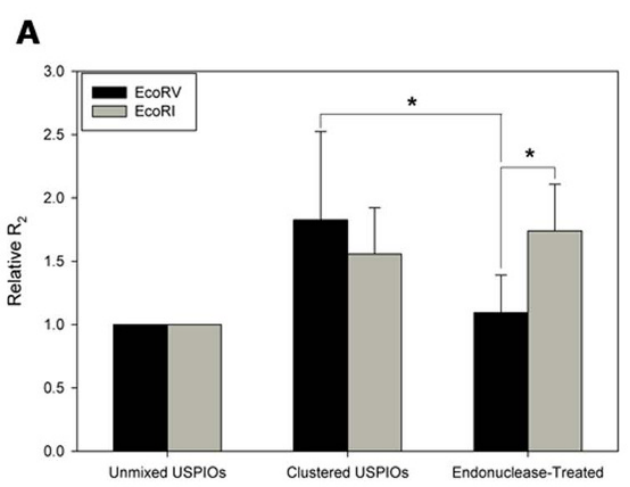

B

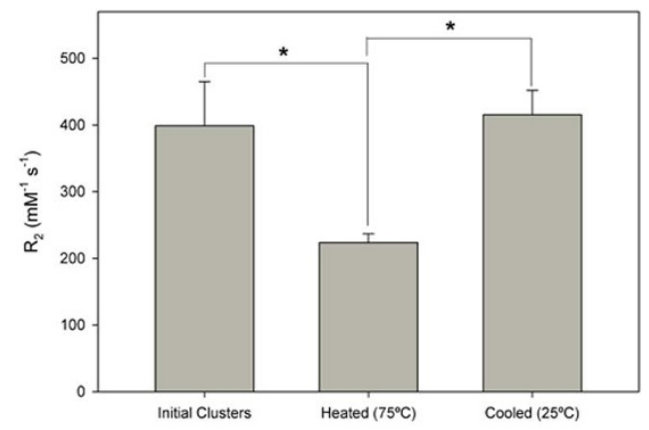

Figure 4 Controlled release of USPIO micelles by environmental triggers. (A) Self-assembly of EcoRV-sensitive ssDNA-USPIO clusters, and subsequent enzymatic treatment results in measurable changes in $R_{2}$ relaxation coefficient relative to initial values. Following EcoRV treatment, $R_{2}$ values return to baseline, a phenomenon that is in significant contrast to the effects of EcoRl treatment of the same clusters $(n=6) .{ }^{*} p<0.05$. (B) Thermocycling of DNA-crosslinked USPIO clusters results in measurable changes in $\mathrm{R}_{2}$ relaxation coefficient. Heating of the clusters melts the DNA and results in declustering of the particles, corresponding to an approximately $50 \%$ decrease in $R_{2}$ coefficient. After allowing the system to cool, the $R_{2}$ coefficient increases to original levels, suggesting the reclustering of the ssDNA-USPIOs $(n=3)$. ${ }^{*} p<0.05$. contrast, clusters were alternatively treated with EcoRI as a negative control, and the lack of a declustering response is reflected in an insignificant change in the $R_{2}$ coefficient of the system.

Next, reversible declustering of the USPIOs was achieved through thermocycling, where $R_{2}$ measurements were made while the USPIO clusters were being subjected to heating and cooling. In this process, heating and cooling of the clusters melts and reanneals the crosslinking DNA sequences, respectively. The resulting changes in the clustering of USPIOs leads to expected fluctuations in $R_{2}$ (Figure $4 \mathrm{~b}$ ). The heated clusters are expected to decluster, resulting in the return of $R_{2}$ values to baseline levels prior to mixing $\mathrm{C} 1$-USPIOs and C2-USPIOs. Allowing the system to cool is expected to reanneal the DNA sequences and reform clusters, returning $R_{2}$ levels to the ranges expected for clusters. Our observations matched these expectations. Heating of the clusters resulted in approximately 50\% decrease in $R_{2}$, while return of the system to room temperature resulted in recovery of the original $R_{2}$.

\section{Conclusions}

Novel PEG-PPS based polymer conjugates were synthesized and characterized, then applied as a USPIO coating in the thin film hydration method to yield USPIO micelles. The synthesis of ssDNA-tagged polymers and the easy incorporation of these species into the micelle formation process leads to the facile formation of USPIO micelles that display biological ligands to the surrounding media. The generation of complementary populations of ssDNA-USPIOs results in a system that is capable of detecting enzymatic cleavage events through significant changes in $R_{2}$ relaxation coefficient of the system. These results motivate ongoing studies in our group involving proteolytically-degradable USPIO clusters for the detection of matrix metalloproteinase activity in tumors.

\section{Methods}

General

All materials and reagents were purchased from SigmaAldrich (St. Louis, MO) and used as purchased unless otherwise specified. Methyl acrylate was purchased from Sigma-Aldrich (St. Louis, MO) and was purified by distillation prior to use. Heterobifunctional PEG reagents were purchased from Laysan Bio (Arab, AL) and used as purchased. The restriction enzymes EcoRI and EcoRV were purchased from New England Biolabs (Ipswich, MA). Custom ssDNA sequences designated C1 (5'amino-ACGTACGTGATATCTGCATGCA-3') and C2 (5'-amino-TGCATGCAGATATCACGTACGT-3') were purchased from Sigma-Genosys. By design, C1 and C2 are complementary sequences that lack the ability to 
self-hybridize into hairpins and other undesired complexes.

Polymer samples were prepared for FT-IR spectroscopy by mixing with IR-grade $\mathrm{KBr}$ and pelleting on a $\mathrm{KBr}$ press. FT-IR was performed on a Bruker Tensor 27 system. ${ }^{1} \mathrm{H}$ NMR spectra were obtained at $400 \mathrm{MHz}$ using a 9.4 T Oxford magnet operated by a Bruker AV400 console. The main NMR probe for the instrument is a $5 \mathrm{~mm} \mathrm{Z}$-gradient broadband inverse (BBI) probe with automatic tuning and matching capability (ATM). Gel permeation chromatography (GPC) was performed on a Tosoh Biosciences TSKGel SuperHZ-M mixed bed column $\left(4 \times 10^{6} \mathrm{Da}\right.$ exclusion limit; DMF $+0.1 \mathrm{M} \mathrm{LiBr}$ mobile phase) incubated at $60^{\circ} \mathrm{C}$ with a Shimadzu SPD10A UV detector and RID-10A refractive index detector (Shimadzu Scientific Instruments, Columbia, MD), and a Wyatt miniDAWN Treos multi-angle light scattering detector (MALS; Wyatt Technology, Santa Barbara, CA).

Transmission electron microscopy (TEM) was conducted on a Philips CM20 system. Carbon film-backed copper grids (Electron Microscopy Sciences, Hatfield, PA) were dipped into nanoparticle suspensions of interest and blotted dry. This process was repeated three times. Images were collected using a CCD camera with AMT Image Capture Engine software (Advanced Microscopy Techniques, Danvers, MA), and sizing of the particles was automated using a particle analyzer on ImageJ software. For nanoparticle micelles deposited from water, samples were dried in a vacuum desiccator for $2 \mathrm{~h}$, and then counterstained with 3\% uranyl acetate in water (Electron Microscopy Sciences, Hatfield, PA) for $30 \mathrm{~s}$, gently blotted dry, and further dried in the vacuum desiccator for another $2 \mathrm{~h}$ prior to imaging.

For thermogravimetric analysis (TGA), samples were weighed as approximately $5 \mathrm{mg}$ and deposited into a platinum pan for analysis with the Instrument Specialist's TGA-1000 (Instrument Specialists, Inc., Twin Lakes, WI). Samples were heated to approximately $200 \mathrm{~K}$ past their expected vaporization point and were heated at a rate of $10 \mathrm{~K}$ per minute.

\section{Synthesis and characterization of oleic acid-coated USPIO cores}

Synthesis of USPIO cores was done based on the procedures described by Woo et al. [14]. Under argon gas flow, oleic acid $(3.8 \mathrm{~mL}, 12 \mathrm{mmol})$ was heated to $100^{\circ} \mathrm{C}$ in $40 \mathrm{~mL}$ octyl ether in a three-neck flask. $\mathrm{Fe}(\mathrm{CO})_{5}$ $(0.8 \mathrm{~mL}, 6 \mathrm{mmol})$ was then injected into the system, and the mixture was then refluxed at $280^{\circ} \mathrm{C}$ for $4 \mathrm{~h}$. Next, the mixture was cooled to $80^{\circ} \mathrm{C}$ and aerated overnight $(>14 \mathrm{~h}$ ). The mixture was then refluxed for $2 \mathrm{~h}$ at $280^{\circ} \mathrm{C}$ and then cooled back to room temperature. Oleic acid-stabilized USPIOs (OA-USPIOs) were collected following three washes in ethanol and centrifugation, and air dried overnight to form a dark brown-black powder.

\section{Synthesis of PPS-COOMe (1)}

The PPS block was synthesized via anionic ring opening polymerization of propylene sulfide from a deprotected ethanethiol initiator (Figure 1). To form the initiator, 3 eq of the deprotectant 1,8-diazabicycloundec-7-ene (DBU; $11.2 \mathrm{~mL} ; 75 \mathrm{mmol}$ ) was mixed in $40 \mathrm{~mL}$ dry DMF in a Schlenk tube, followed by the addition of 1 eq of ethanethiol $(1.85 \mathrm{~mL} ; 25 \mathrm{mmol})$. The tube was evacuated via a membrane pump and equilibrated with argon $6 \times$, and then stirred at room temperature for $10 \mathrm{~min}$. Monomer was then added to the vessel by injection of 10 eq propylene sulfide $(19.6 \mathrm{~mL} ; 250 \mathrm{mmol})$ into the vial, and polymerization occurred for $90 \mathrm{~min}$. In a separate Schlenk tube, 10 eq distilled methyl acrylate $(22.5 \mathrm{~mL} ; 250 \mathrm{mmol})$ was mixed with 5 eq triethylamine $\left(\mathrm{Et}_{3} \mathrm{~N} ; 17.4 \mathrm{~mL} ; 125 \mathrm{mmol}\right)$. This vial was evacuated via a membrane pump and equilibrated with argon gas $6 \times$, and then the contents were transferred under vacuum into the PPS-containing vial. Upon mixing of the two liquids, a color change is observed from orange to yellowish. This mixture was then left to stir overnight at room temperature. Concentrated product was obtained by removal of DMF under high vacuum, and was redissolved in $\mathrm{CH}_{2} \mathrm{Cl}_{2}(100 \mathrm{~mL})$. This solution was extracted 7 times in brine. The collected organic phase was then dried over $5 \mathrm{~g}$ of sodium sulfate, and residual salts were removed by gravity filtration through a \#5 Whatman filter disc. The product was concentrated by incomplete evaporation of the $\mathrm{CH}_{2} \mathrm{Cl}_{2}$ under vacuum, and then precipitated by addition to ice-cold hexanes for $30 \mathrm{~min}$. Centrifugation for $5 \mathrm{~min}$ at $800 \times \mathrm{g}$ pellets the PPS block, and the hexane extraction step and centrifugation was repeated a second time to yield $\mathrm{PPS}_{10}$-COOMe (PPS-COOMe). Average degree of polymerization was estimated by NMR. FT-IR ( $\mathrm{KBr}) 1737$ (s, ester $\mathrm{C}=\mathrm{O}$ ), 1490-1400 ( $\mathrm{t}, \mathrm{C}-\mathrm{H}$ from PPS block and ethyl terminus overlapped), $693\left(\mathrm{~s}, \mathrm{CH}_{2}-\mathrm{S}\right) . \delta_{\mathrm{H}}\left(400 \mathrm{MHz} ; \mathrm{CDCl}_{3}\right): \delta$ 1.2-1.3 (t, $\mathrm{CH}_{2}$ next to carboxylic terminus), 1.3-1.4 (d, $\mathrm{CH}_{3}$ in PPS block \& terminal $\mathrm{CH}_{3}$ ), 2.5-2.8 (broad s, $\mathrm{CH}$ in PPS block), 2.8-3.1 (broad s, $\mathrm{CH}_{2}$ next to $\mathrm{S}$ ), 3.72 (s, $\mathrm{CH}_{3}$ in ester).

\section{Synthesis of PPS-COOH (2)}

$\mathrm{PPS}_{10}-\mathrm{COOH}$ was synthesized from $\mathrm{PPS}_{10}$-COOMe (1) by mixing the latter in $0.1 \mathrm{M} \mathrm{NaOH}$ in $\mathrm{DMF}$ at $65^{\circ} \mathrm{C}$ for $5 \mathrm{~h}$ under open air in a fume hood (Figure 1). This setup drives the reaction forward as the $\mathrm{MeOH}$ byproduct evaporates directly into the environment. After the reaction was cooled to room temperature, concentrated 
product was obtained by evaporation of DMF under high vacuum, and was redissolved in $\mathrm{CH}_{2} \mathrm{Cl}_{2}(100 \mathrm{~mL})$. This solution was extracted 7 times in brine. The collected organic phase was then dried over $5 \mathrm{~g}$ of sodium sulfate, and residual salts were removed by gravity filtration through a \#5 Whatman filter disc. The product was concentrated by incomplete evaporation of the $\mathrm{CH}_{2} \mathrm{Cl}_{2}$ under vacuum, and then precipitated by addition to icecold hexanes for $30 \mathrm{~min}$. Centrifugation for $5 \mathrm{~min}$ at $800 \times \mathrm{g}$ pellets the PPS block, and the hexane extraction step and centrifugation was repeated a second time to yield $\mathrm{PPS}_{10}-\mathrm{COOH}$ (PPS-COOH), a viscous yellow liquid, at more than $90 \%$ conversion, as confirmed by NMR spectroscopy. The carboxylic acid terminus remains unprotonated, as confirmed by the lack of the corresponding proton peak on FT-IR and NMR. FT-IR ( $\mathrm{KBr}) 1737$ (s, ester $\mathrm{C}=\mathrm{O}$; incomplete ester hydrolysis), 1713 (s, carboxylic $\mathrm{C}=\mathrm{O}$ ), 1490-1400 (t, $\mathrm{C}-\mathrm{H}_{3}$ and C$\mathrm{H}_{2}$ overlapped), 693 (s, $\left.\mathrm{CH}_{2}-\mathrm{S}\right) . \delta_{H}\left(400 \mathrm{MHz} ; \mathrm{CDCl}_{3}\right): \delta$ 1.2-1.3 ( $\mathrm{t}, \mathrm{CH}_{2}$ next to carboxylic terminus), 1.3-1.4 (d, $\mathrm{CH}_{3}$ in PPS block \& terminal $\mathrm{CH}_{3}$ ), 2.5-2.8 (broad s, $\mathrm{CH}$ in PPS block), 2.8-3.1 (broad s, $\mathrm{CH}_{2}$ next to $\mathrm{S}$ ).

\section{Synthesis of cPEG-PPS (carboxylated PEG-PPS; 3) and PPS- PEG-ssDNA conjugates}

$\mathrm{PPS}_{10}-\mathrm{COOH}(2), 2 \mathrm{~g}$ was dissolved into $3 \mathrm{~mL}$ of $\mathrm{CH}_{2} \mathrm{Cl}_{2}$ and reacted with $\sim 5$ eq of $N$-hydroxysuccinimide (NHS; 1.44 g; $12.5 \mathrm{mmol}$ ), 1-Ethyl-3-(3-dimethylaminopropyl) carbodiimide hydrochloride (EDC; $2.40 \mathrm{~g}$; $12.5 \mathrm{mmol})$, and $\mathrm{Et}_{3} \mathrm{~N}(1.74 \mathrm{~mL} ; 12.5 \mathrm{mmol})$ with gentle vortexing for $4 \mathrm{~h}$ at room temperature. Following the reaction, the crude product was concentrated by rotary evaporation. Excess salts were precipitated and extracted $2 \times$ with brine and $3 \times$ with deionized water, and the product was dried by rotary evaporation. $1 \mathrm{~mL}$ of the product was redissolved in $5 \mathrm{~mL}$ of DMF, and then reacted with $\sim 0.1$ eq of $M_{n} 5 \mathrm{kDa} \mathrm{H}{ }_{2} \mathrm{~N}$-PEG-COOH $(625 \mathrm{mg}$; $\sim 125 \mu \mathrm{mol})$ in the presence of 0.2 eq of $\mathrm{Et}_{3} \mathrm{~N}(34 \mu \mathrm{L}$; $250 \mu \mathrm{mol}$ ) overnight. The crude product was concentrated by rotary evaporation, dissolved in $10 \mathrm{~mL}$ $\mathrm{CH}_{2} \mathrm{Cl}_{2}$, and precipitated twice in diethyl ether under ice for $1 \mathrm{~h}$ in order to remove unreacted PPS. Excess organic solvents were removed by rotary evaporation, and the crude product was dissolved in deionized water and rinsed in $100 \mathrm{kDa}$ MWCO centrifugal filters (Corning Life Sciences, Lowell, MA) with six fill volumes of deionized water, to remove unbound PEG. Lyophilization of the product overnight yielded cPEG-PPS (carboxylated PEG-PPS). $\delta_{\mathrm{H}}\left(400 \mathrm{MHz} ; \mathrm{CDCl}_{3}\right): \delta 1.2-1.3(\mathrm{t}$, $\mathrm{CH}_{2}$ next to carboxylic terminus), 1.3-1.4 (d, $\mathrm{CH}_{3}$ in PPS block \& terminal $\mathrm{CH}_{3}$ ), 1.4-1.8 (broad s, $\mathrm{CH}_{2}$ next to $\mathrm{COOH}$ ), 2.5-2.8 (broad s, CH in PPS block), 2.8-3.1 (broad s, $\mathrm{CH}_{2}$ next to $\mathrm{S}$ ), 3.6-3.8 (s, $\mathrm{CH}_{2}$ in PEG block), 4.18 (s, NH in amide bond).
To construct the ssDNA-PEG-PPS conjugates, the 5'aminated custom ssDNA sequences $\mathrm{C} 1$ and $\mathrm{C} 2$ were each separately reacted with cPEG-PPS in $5 \mathrm{~mL}$ sequencing grade DMF in scintillation vials. $1.5 \mu \mathrm{mol}$ of each ssDNA sequence was transferred to each DMFcontaining vial in $0.5 \mathrm{~mL} \mathrm{NaCl}$ buffer in water. Following addition of equimolar amounts of cPEG-PPS, 5 eq of $\mathrm{EDC}$ and $\mathrm{Et}_{3} \mathrm{~N}$ were added to the reactions. The mixtures were briefly bubbled with argon gas, then capped and vortexed for $2 \mathrm{~h}$ at room temperature. Following rotary evaporation to remove excess $\mathrm{DMF}$ and $\mathrm{Et}_{3} \mathrm{~N}$, the crude products were dissolved in DNAse-free water (Sigma-Aldrich) and dialyzed separately in $30 \mathrm{kDa}$ MWCO centrifugal filters (Corning Life Sciences, Lowell, MA) with ten fill volumes of DNAse-free water. The presence of DNA-polymer conjugates was confirmed by the appearance of a $260 \mathrm{~nm}$ absorbance peak versus unreacted cPEG-PPS, by UV-Vis spectrophotometry.

\section{Encapsulation of USPIO core nanoparticles with polymers}

USPIO-core, polymer-shell micelles/nanoparticles were formed by the thin-film hydration method [39]. Briefly, $15 \mathrm{mg}$ of purified PEG-PPS-based polymers were dissolved with $10 \mathrm{mg}$ of OA-USPIOs in $1 \mathrm{~mL}$ toluene, vortexed to mix, sonicated for $5 \mathrm{~s}$ to break apart clumps, and then dried by rotary evaporation for $20 \mathrm{~min}$. The dried polymer/USPIO mixture was then rehydrated in $5 \mathrm{~mL}$ of DNAse-free water and vortexed vigorously to suspend all particulates. Large clumps and byproducts are easily removed by magnetic pelleting, and the colloidal phase is collected and further centrifuged at $2500 \times$ $\mathrm{g}$ for $5 \mathrm{~min}$ to precipitate excess polymers. The supernatant is gently aspirated by pipet into fresh scintillation vials and stored at $4^{\circ} \mathrm{C}$.

\section{Phenanthroline assay for iron content determination}

To quantify the concentration of iron in all PEG-PPSUSPIO formulations, the 1,10-phenanthroline assay was used [44]. USPIOs in PBS $(50 \mu \mathrm{L})$ were mineralized by treatment in concentrated $\mathrm{H}_{2} \mathrm{SO}_{4}$ for 30 min at room temperature, resulting in a loss of the dark brownishblack color of the solution. This was followed by treatment of the mixture with $10 \mu \mathrm{L} 100 \mathrm{mg} / \mathrm{mL}$ hydroxylammonium chloride in water and $50 \mu \mathrm{L} 1 \mathrm{mg} / \mathrm{mL}$ 1,10-phenanthroline in water. Development of an intense orange color, corresponding to the presence of iron, is observed upon addition of $550 \mu \mathrm{L} 100 \mathrm{mg} / \mathrm{mL}$ sodium acetate in water. Absorbance at $510 \mathrm{~nm}$ was measured on a Varian Cary 50 UV-Vis-NIR spectrophotometer (Palo Alto, CA). The concentration of free iron was calculated based on a standard curve constructed using serial dilutions of ferrous ammonium sulfate (Fisher Scientific, Pittsburgh, PA) in water. Measurements of each sample were done in triplicate. 


\section{R2 relaxation measurements}

A 0.5 T Maran tabletop NMR scanner with DRX-II console (Oxford Instruments, Oxfordshire, UK) was used for transverse $\left(\mathrm{T}_{2}\right)$ relaxation time measurements. 200 $\mu \mathrm{L}$ of PEG-PPS-USPIOs in PBS were loaded into 5-mm thin-walled NMR tubes and introduced into the scanner. Measurements were made using a Carr-Purcell-Meiboom-Gill (CPMG) sequence at room temperature, 32 echoes with $12 \mathrm{~ms}$ time between echoes, and an average of 9 acquisitions. $R_{2}$ relaxation coefficients were calculated based on the following formula [46], where [Fe] is the iron content of the sample as determined through the phenanthroline assay (described earlier):

$$
R_{2}=\frac{1}{T_{2} \times[\mathrm{Fe}]}
$$

For clustering/declustering experiments, $100 \mu \mathrm{L}$ of complementary ssDNA-USPIO populations were mixed in the NMR tubes and allowed $10 \mathrm{~min}$ to cluster before $\mathrm{T}_{2}$ was remeasured as described above. To study the effects of restriction enzyme treatment, $500 \mathrm{U}$ of EcoRI or EcoRV were added to the tubes according to the manufacturer's instructions and the system was incubated at $37^{\circ} \mathrm{C}$ for $4 \mathrm{~h}$ before relaxation time was remeasured. To study the effects of thermocycling, samples in NMR tubes were heated to $85^{\circ} \mathrm{C}$ in a water bath for $15 \mathrm{~min}$, then measured in the relaxometer. The temperatures in heated ssDNA-USPIO samples did not drop below $70^{\circ} \mathrm{C}$ during the measurement process. Unless otherwise noted, all presented data is the average of three independent experiments. Statistical significance was established using the paired Student's t-test for all samples.

\section{Acknowledgements \\ This work was supported by a grant from the Department of Defense Congressionally Directed Medical Research Programs (W81XWH-08-1-0502). Dynamic light scattering, spectrofluorimetry, and TEM were conducted through the use of the core facilities of the Vanderbilt Institute of Nanoscale Sciences and Engineering (VINSE). Mass spectrometry was conducted in the VUMC Mass Spectrometry Core, and the authors thank M. Wade Calcutt for extensive technical support and discussions. Relaxation measurements were made possible through the facilities of the Vanderbilt University Institute of Imaging Science (VUIIS). The authors would also like to thank Darrell Morgan of Corning Life Sciences for providing the centrifugal filters/concentrators used in this study.}

\section{Author details}

${ }^{1}$ Department of Biomedical Engineering, Vanderbilt University; Nashville, Tennessee, USA. ${ }^{2}$ Vanderbilt Institute for Nanoscale Science and Engineering, Vanderbilt University; Nashville, Tennessee, USA. ${ }^{3}$ Interdisciplinary Program in Materials Science, Vanderbilt University; Nashville, Tennessee, USA.

${ }^{4}$ Integrative Biosciences Institute, École Polytechnique Fédérale de Lausanne, Lausanne, Switzerland.

\section{Authors' contributions}

SSY and RLS planned and carried out all polymer synthesis, characterization, and micelle synthesis and characterization, with extensive input from TDG. $\mathrm{RAO}$ carried out all gravimetric work and repeated relaxation measurements.
CSB performed all NMR work and aided in analysis. CPO and JAH contributed extensive technical consultation and expertise on the properties and synthesis of the block copolymers used in this study. All authors have read and approved the final manuscript.

\section{Competing interests}

The authors declare that they have no competing interests.

Received: 11 November 2010 Accepted: 27 February 2011 Published: 27 February 2011

\section{References}

1. Scherer RL, McIntyre JO, Matrisian LM: Imaging matrix metalloproteinases in cancer. Cancer Metastasis Rev 2008, 27:679-690.

2. Galis ZS, Khatri JJ: Matrix metalloproteinases in vascular remodeling and atherogenesis: the good, the bad, and the ugly. Circ Res 2002, 90:251-262.

3. Thornton PD, Heise A: Highly specific dual enzyme-mediated payload release from peptide-coated silica particles. J Am Chem Soc 2010, 132:2024-2028.

4. Venkatesh S, Wower J, Byrne ME: Nucleic acid therapeutic carriers with on-demand triggered release. Bioconjug Chem 2009, 20:1773-1782.

5. Schlossbauer A, Kecht J, Bein T: Biotin-avidin as a protease-responsive cap system for controlled guest release from colloidal mesoporous silica. Angew Chem Int Ed Engl 2009, 48:3092-3095.

6. Chang E, Miller JS, Sun J, Yu WW, Colvin VL, Drezek R, West JL: Proteaseactivated quantum dot probes. Biochem Biophys Res Commun 2005, 334:1317-1321.

7. Mclntyre JO, Fingleton B, Wells KS, Piston DW, Lynch CC, Gautam S, Matrisian LM: Development of a novel fluorogenic proteolytic beacon for in vivo detection and imaging of tumour-associated matrix metalloproteinase-7 activity. Biochem J 2004, 377:617-628.

8. Jeong U, Teng XW, Wang Y, Yang H, Xia YN: Superparamagnetic colloids: Controlled synthesis and niche applications. Advanced Materials 2007, 19:33-60.

9. Josephson L, Tung CH, Moore A, Weissleder R: High-efficiency intracellular magnetic labeling with novel superparamagnetic-Tat peptide conjugates. Bioconjug Chem 1999, 10:186-191.

10. Park JH, von Maltzahn G, Ruoslahti E, Bhatia SN, Sailor MJ: Micellar hybrid nanoparticles for simultaneous magnetofluorescent imaging and drug delivery. Angew Chem Int Ed Engl 2008, 47:7284-7288.

11. Perez JM, Josephson $L$, Weissleder R: Use of magnetic nanoparticles as nanosensors to probe for molecular interactions. Chembiochem 2004, $5: 261-264$

12. Cormode DP, Skajaa T, Fayad ZA, Mulder WJ: Nanotechnology in medical imaging: probe design and applications. Arteriosclerosis, thrombosis, and vascular biology 2009, 29:992-1000.

13. Cormode DP, Briley-Saebo KC, Mulder WJ, Aguinaldo JG, Barazza A, Ma Y, Fisher EA, Fayad ZA: An ApoA-I mimetic peptide high-density-lipoproteinbased MRI contrast agent for atherosclerotic plaque composition detection. Small 2008, 4:1437-1444.

14. Woo K, Hong J, Choi S, Lee HW, Ahn JP, Kim CS, Lee SW: Easy synthesis and magnetic properties of iron oxide nanoparticles. Chemistry of Materials 2004, 16:2814-2818.

15. Xie J, Peng S, Brower N, Pourmand N, Wang SX, Sun SH: One-pot synthesis of monodisperse iron oxide nanoparticles for potential biomedical applications. Pure and Applied Chemistry 2006, 78:1003-1014.

16. Kohler N, Fryxell GE, Zhang M: A bifunctional poly(ethylene glycol) silane immobilized on metallic oxide-based nanoparticles for conjugation with cell targeting agents. J Am Chem Soc 2004, 126:7206-7211.

17. Talelli M, Rijcken CJ, Lammers T, Seevinck PR, Storm G, van Nostrum CF, Hennink WE: Superparamagnetic iron oxide nanoparticles encapsulated in biodegradable thermosensitive polymeric micelles: toward a targeted nanomedicine suitable for image-guided drug delivery. Langmuir 2009, 25:2060-2067.

18. Nitin N, LaConte LE, Zurkiya O, Hu X, Bao G: Functionalization and peptide-based delivery of magnetic nanoparticles as an intracellular MRI contrast agent. J Biol Inorg Chem 2004, 9:706-712.

19. Hermanson GT: Bioconjugate Techniques. San Diego: Academic Press; 2 2008.

20. Briley-Saebo KC, Mani V, Hyafil F, Cornily JC, Fayad ZA: Fractionated Feridex and positive contrast: in vivo MR imaging of atherosclerosis. Magn Reson Med 2008, 59:721-730. 
21. Raynal I, Prigent P, Peyramaure S, Najid A, Rebuzzi C, Corot C: Macrophage endocytosis of superparamagnetic iron oxide nanoparticles: mechanisms and comparison of ferumoxides and ferumoxtran-10. Invest Radiol 2004, 39:56-63.

22. Bonnemain B: Superparamagnetic Agents in Magnetic Resonance Imaging: Physicochemical Characteristics and Clinical Applications A Review. Journal of Drug Targeting 1998, 6:167-174.

23. Tan YF, Chandrasekharan P, Maity D, Yong CX, Chuang KH, Zhao Y, Wang S, Ding J, Feng SS: Multimodal tumor imaging by iron oxides and quantum dots formulated in poly (lactic acid)-d-alpha-tocopheryl polyethylene glycol 1000 succinate nanoparticles. Biomaterials 2011.

24. Foy SP, Manthe RL, Foy ST, Dimitrijevic S, Krishnamurthy N, Labhasetwar V: Optical imaging and magnetic field targeting of magnetic nanoparticles in tumors. ACS Nano 2010, 4:5217-5224.

25. Pouliquen D, Lucet I, Chouly C, Perdrisot R, Le Jeune JJ, Jallet P: Liverdirected superparamagnetic iron oxide: quantitation of $\mathrm{T} 2$ relaxation effects. Magn Reson Imaging 1993, 11:219-228

26. Pochon S, Hyacinthe R, Terrettaz J, Robert F, Schneider M, Tournier H: Long circulating superparamagnetic particles with high $\mathrm{T} 2$ relaxivity. Acta Radiol Supp/ 1997, 412:69-72.

27. Kim BS, Qiu JM, Wang JP, Taton TA: Magnetomicelles: composite nanostructures from magnetic nanoparticles and cross-linked amphiphilic block copolymers. Nano Lett 2005, 5:1987-1991.

28. Ai H, Flask C, Weinberg B, Shuai X, Pagel MD, Farrell D, Duerk J, Gao JM: Magnetite-loaded polymeric micelles as ultrasensitive magneticresonance probes. Advanced Materials 2005, 17:1949-+.

29. Napoli A, Tirelli N, Wehrli E, Hubbell JA: Lyotropic behavior in water of amphiphilic ABA triblock copolymers based on poly(propylene sulfide) and poly(ethylene glycol). Langmuir 2002, 18:8324-8329.

30. Napoli A, Valentini M, Tirelli N, Muller M, Hubbell JA: Oxidation-responsive polymeric vesicles. Nat Mater 2004, 3:183-189.

31. Rehor A, Hubbell JA, Tirelli N: Oxidation-sensitive polymeric nanoparticles. Langmuir 2005, 21:411-417.

32. Segura T, Hubbell JA: Synthesis and in vitro characterization of an $A B C$ triblock copolymer for siRNA delivery. Bioconjug Chem 2007, 18:736-745.

33. Perez JM, Josephson L, O'Loughlin T, Hogemann D, Weissleder R: Magnetic relaxation switches capable of sensing molecular interactions. Nat Biotechnol 2002, 20:816-820.

34. Cerritelli S, Velluto D, Hubbell JA: PEG-SS-PPS: reduction-sensitive disulfide block copolymer vesicles for intracellular drug delivery. Biomacromolecules 2007, 8:1966-1972.

35. Napoli A, Tirelli N, Kilcher G, Hubbell JA: New synthetic methodologies for amphiphilic multiblock copolymers of ethylene glycol and propylene sulfide. Macromolecules 2001, 34:8913-8917.

36. O'Neil CP, Suzuki T, Demurtas D, Finka A, Hubbell JA: A novel method for the encapsulation of biomolecules into polymersomes via direct hydration. Langmuir 2009, 25:9025-9029.

37. O'Neil CP, van der Vlies AJ, Velluto D, Wandrey C, Demurtas D, Dubochet J, Hubbell JA: Extracellular matrix binding mixed micelles for drug delivery applications. J Control Release 2009, 137:146-151.

38. Rehor A, Tirelli N, Hubbell JA: A new living emulsion polymerization mechanism: Episulfide anionic polymerization. Macromolecules 2002, 35:8688-8693.

39. Dubertret $B$, Skourides $P$, Norris DJ, Noireaux V, Brivanlou AH, Libchaber A: In vivo imaging of quantum dots encapsulated in phospholipid micelles. Science 2002, 298:1759-1762.

40. Yu WW, Chang E, Falkner JC, Zhang J, Al-Somali AM, Sayes CM, Johns J, Drezek R, Colvin VL: Forming biocompatible and nonaggregated nanocrystals in water using amphiphilic polymers. J Am Chem Soc 2007, 129:2871-2879

41. Yu WW, Chang E, Sayes CM, Drezek R, Colvin VL: Aqueous dispersion of monodisperse magnetic iron oxide nanocrystals through phase transfer. Nanotechnology 2006, 17:4483-4487.

42. Laurent S, Forge D, Port M, Roch A, Robic C, Vander Elst L, Muller RN: Magnetic iron oxide nanoparticles: synthesis, stabilization, vectorization, physicochemical characterizations, and biological applications. Chem Rev 2008, 108:2064-2110

43. Zhang L, He R, Gu HC: Oleic acid coating on the monodisperse magnetite nanoparticles. Applied Surface Science 2006, 253:2611-2617.

44. Christian GD: Analytical chemistry. New York: Wiley \& Sons; 51994
45. Lee JH, Huh YM, Jun YW, Seo JW, Jang JT, Song HT, Kim S, Cho EJ, Yoon HG, Suh JS, Cheon J: Artificially engineered magnetic nanoparticles for ultra-sensitive molecular imaging. Nat Med 2007, 13:95-99.

46. LaConte LEW, Nitin N, Zurkiya O, Caruntu D, O'Connor CJ, Hu XP, Bao G: Coating thickness of magnetic iron oxide nanoparticles affects R-2 relaxivity. Journal of Magnetic Resonance Imaging 2007, 26:1634-1641.

doi:10.1186/1477-3155-9-7

Cite this article as: Yu et al.: Enzymatic- and temperature-sensitive controlled release of ultrasmall superparamagnetic iron oxides (USPIOs). Journal of Nanobiotechnology 2011 9:7.

\section{Submit your next manuscript to BioMed Central and take full advantage of:}

- Convenient online submission

- Thorough peer review

- No space constraints or color figure charges

- Immediate publication on acceptance

- Inclusion in PubMed, CAS, Scopus and Google Scholar

- Research which is freely available for redistribution 